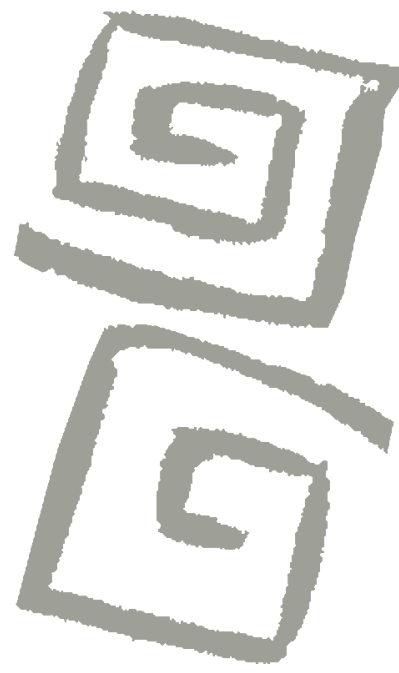

\title{
Antipsicóticos para la esquizofrenia: paradigma de los medicamentos psiquiátricos
}

\author{
Antipsychotics for schizophrenia: the paradigm of \\ psychiatric drugs
}

Pol Yanguas, Emilio'

${ }^{1}$ Doctor en Farmacia. Jefe, Unidad de Farmacia, Centro Dr. Esquerdo para Enfermos Mentales, Diputación Provincial de Alicante, España. epol@diputacionalicante.es
RESUMEN Los antipsicóticos no parecen revertir las causas de la esquizofrenia y, aunque son fármacos que pueden aliviar los síntomas a corto y mediano plazo, a largo plazo pueden no ser beneficiosos e incluso ser contraproducentes. Su empleo debería limitarse a situaciones agudas con agitación y tensión incapacitante. Presentan considerables efectos adversos y, ante la negativa de una persona a seguir tomándolos, adoptar una estrategia de reducción de daños apoyando y supervisando la retirada puede ser preferible a la coerción. Existen alternativas a los neurolépticos. Los prescriptores deberían estar más atentos y considerar las valoraciones que los usuarios hacen de sus efectos. El apego a las guías de tratamiento es escaso, seguramente por basarse en ensayos clinicos de calidad deficente, que deben mejorar y prolongarse en el tiempo. La raíz del problema probablemente se encuentra en la tautología sobre la etiología y naturaleza biológica de lo que Ilaman esquizofrenia, que realmente no parece ser más que un constructo ideológico-comercial.

PALABRAS CLAVES Esquizofrenia; Antipsicóticos; Mercadeo; Efectividad; Cumplimiento de la Medicación.

ABSTRACT Antipsychotic drugs do not appear to reverse the causes of schizophrenia, and although they can relieve symptoms in the short to medium term, in the long term they may not be beneficial and could even be counterproductive. Their use should be limited to acute situations in which agitation and tension is disabling. The drugs have significant adverse effects, and given the refusal of a person to continue taking them, a harm reduction strategy to support and monitor the withdrawal may be preferable to coercion. There are alternatives to neuroleptics. Prescribers should be more vigilant and consider the assessments of users regarding the drugs' effects. Adherence to treatment guidelines is low, probably because the guidelines are based on clinical trials of deficient quality which consequently should be improved and extended over a greater period of time. The root of the problem is likely the tautology on the etiology and biological nature of what is known as schizophrenia, which in fact does not seem to be more than a commercial and ideological construct.

KEY WORDS Schizophrenia; Antipsychotic Agents; Marketing; Effectiveness; Medication Adherence. 


\section{INTRODUCCIÓN}

Muchas medicinas son azules, entre ellas, el Haldol. Yo tomo Haldol para no tener ninguna ilusión de que moriré loco, un día, en un lugar sucio y sin comida. Es el fin de cualquier loco. (Todos los perros son azules, Rodrigo de Souza Leão)

La esquizofrenia se caracteriza por dificultades para diferenciar lo real de lo irreal. Se acompaña de ansiedad, depresión y, ocasionalmente, conductas suicidas. Los que la padecen sienten tensión, dificultades de concentración, insomnio, alucinaciones auditivas y/o visuales, mantienen creencias falsas, el pensamiento y el afecto aparecen alterados. Es una importante causa de discapacidad a largo plazo, altera las relaciones sociales y familiares, conlleva dificultades educativas y ocupacionales, pérdida de productividad, desempleo, enfermedad física y mortalidad prematura. Se postula una base biológica, consistente en hiperfunción dopaminérgica, debida a una susceptibilidad particular de origen genético $y$, en algún caso, infecciosa, tóxica o traumática. Sin embargo, para su manifestación, son fundamentales los factores biográficos y las dificultades psicosociales (1).

Los Ilamados antipsicóticos se consideran la clave del tratamiento de la esquizofrenia (2). Esta denominación indica que actúan específicamente sobre los procesos neuroquímicos alterados que causan la psicosis; aunque también se nombran con términos menos específicos: tranquilizantes mayores (que calman), ataráxicos (que inducen imperturbabilidad) o neurolépticos (que sujetan los nervios).

Poco después de la introducción de los neurolépticos en clínica se observó que actuaban como antagonistas sobre los receptores de dopamina. Su potencia clínica se correlacionó con la afinidad por estos receptores. Surgió la hipótesis dopaminérgica de la esquizofrenia (3). Si los antagonistas dopaminérgicos mejoraban la esquizofrenia, una hiperactividad dopaminérgica estaría implicada en su fisiopatología. Se conocía que las anfetaminas incrementaban la liberación de dopamina e inducían estados esquizofreniformes y podían agravar los preexistentes, y estos estados pudieron tratarse con neurolépticos. Estudios post mortem indicaban una sobreexpresión de receptores depaminérgicos en el cerebro de sujetos esquizofrénicos, aunque esto podría explicarse por el uso previo de neurolépticos. Esta sobreexpresión también se observó con técnicas de neuroimagen in vivo en sujetos esquizofrénicos no expuestos a neurolépticos, pero no consistentemente (4). La vía dopaminérgica nigroestriada se asoció al control motor, la vía mesolímbica al control de conductas complejas y sistema motivacional, mientras que la vía mesocortical se asoció con la función cognitiva y la respuesta a presiones y dificultades del entorno, la vía tuberoinfundibular interviene en la regulación neuroendocrina. Estas áreas conductuales tradicionalmente se habían relacionado con la esquizofrenia. No obstante, había una disociación temporal entre efectos antidopaminérgicos terapéuticos y adversos. Además, era dificultoso correlacionar la hiperactividad dopaminérgica con la psicopatología de la esquizofrenia, entonces se postuló que la hiperactividad dopaminérgica subcortical explicaría los síntomas positivos de la esquizofrenia (agitación, alucinaciones y delirios), mientras que una hipoactividad frontal explicaría los negativos (apatía, abulia, desinterés).

Como el uso clínico de los antipsicóticos no se limita a la esquizofrenia, y se han utilizado inespecíficamente para el tratamiento de trastornos muy diversos, una nueva hipótesis dopaminérgica convierte a este neurotransmisor en la vía final común en el desarrollo de psicosis y otros trastornos, interviniendo en un juego de contrapesos entre varios neurotransmisores que al desequilibrarse predisponen a los trastornos mentales. El incremento de la actividad dopaminérgica puede ser previo a la psicosis, en respuesta a cambios ambientales (5).

Varias anomalías anatómicas cerebrales se han asociado con la esquizofrenia como el agrandamiento ventricular, la atrofia prefrontal y otras; pero carecen de sensibilidad y especificidad adecuadas para tener utilidad diagnóstica, dado que se encuentran solo entre el $30 \%$ y el $40 \%$ de los sujetos afectados, y también entre el $10 \%$ y el $30 \%$ de los controles (1). Podrían estar causadas por situaciones de presión y marginación en etapas claves del desarrollo (6).

Como no se pueden identificar marcadores biológicos en la esquizofrenia, el diagnóstico se 
basa en las quejas y conductas de los pacientes y su evolución temporal. Esta información se compara con los criterios diagnósticos, establecidos por consenso en comités de clínicos expertos. El $83 \%$ de los miembros del panel para trastornos psicóticos de la cuarta edición del Diagnostic and Statistical Manual of Mental Disorders (DSM-IV) recibía financiación por parte de la industria farmacéutica (7). Las categorías diagnósticas en los trastornos mentales no son realidades objetivas, sino meras convenciones con notable carga ideológica. Aunque la denominación actual de esquizofrenia procede del siglo XIX y había sido descrita desde la antigüedad, sigue sin definirse satisfactoriamente (8), lo que permite la manipulación de datos. La proporción de mejorías en la esquizofrenia durante el periodo 1895-1955 fue del 35,4\%, y en el periodo 1956-1985 fue del 48,5\%. Este aumento se atribuyó al descubrimiento de clorpromazina, pero también coincidió con una relajación de los criterios diagnósticos. Con la vuelta a criterios más estrictos en en la década de 1970 y 1980, la tasa de mejorías retornó al 36,4\% (9).

\section{EL ENORME ÉXITO DE LOS NEUROLÉPTICOS}

El consumo de neurolépticos en España aumentó un 540\% entre 1985 y 2006 (10). En Australia, entre 2000 y 2011 aumentó el 217,7\% (11). En EE.UU., a lo largo de los años, se encuentran varios neurolépticos entre los fármacos líderes en ventas (12). En Manitoba, Canadá, la venta de neurolépticos se incrementó en un $227 \%$, con un aumento de usuarios del $62 \%$ entre 1996 y 2006 (13).

El crecimiento de las ventas de neurolépticos refleja varias estrategias de promoción simultáneas: aumento de las indicaciones autorizadas, deslizamiento de los límites diagnósticos mediante el artificio de "trastornos del espectro...", o definiendo como patológico lo normal, promoviendo un uso inespecífico para trastornos de conducta, expandiendo el uso a poblaciones como ancianos, discapacitados intelectuales, niños y adolescentes. Otra estrategia es promover el uso de dosis superiores a las máximas recomendadas oficialmente, problema que se agrava con la polifarmacia.
A mediados del siglo XX se desató una oleada de optimismo terapéutico relacionada con la llegada de la clorpromazina, que no podía atribuirse a pruebas decisivas sobre su eficacia. En esa época los tratamientos psicodinámicos se habían abandonado y los tratamientos físico-psiquiátricos disponibles resultaban crueles. Los tranquilizantes comercializados en ese momento (barbitúricos, carbamatos, bromuros) presentaban dificultades de manejo: excitación paradójica previa a la sedación; dosis letal muy próxima a la terapéutica; posibilidad de precipitar confusión y psicosis toxicas, y elevado riesgo de dependencia; abuso y reacciones por retirada. Estas no parecían presentarse con clorpromazina, y al comprobarse su capacidad tranquilizante, sustituyó rápidamente a sus antecesores (14).

El éxito de la llegada de clorpromazina al mercado farmacéutico norteamericano fue enorme, en 1955 la empresa SmithKline\&French facturó 75 millones de dólares (15). Ese año, tres de cada diez prescripciones eran de tranquilizantes $y$, en el estado de Nueva York, eran una de cada dos (16). La bibliografía distribuida por la industria de medicamentos entre la profesión médico-farmacéutica era engañosa en dos aspectos. Primero, presentaba el medicamento de forma que conducía a un uso indiscriminado para casi cualquier problema, estado emocional o etapa de la vida, profesión y/o circunstancia. Segundo, hacía poca o ninguna mención sobre los efectos adversos, contraindicaciones y uso a largo plazo, a lo sumo indicaban que seguían investigando, induciendo falsa seguridad. La prensa profesional y general daba a entender que se había logrado la "aspirina psiquiátrica", la "píldora de la felicidad" que calmaba el dolor mental y emocional y eliminaba la psicopatología.

En 1957, el artículo de Brill y Patton (17) informaba una expansión del uso de tranquilizantes (clorpromazina, principalmente) del orden del 250\% durante 1955 y 1956, en los frenopáticos del estado de New York. Paralelamente, hubo un descenso de 500 internos y otros tantos entre 1956 y 1957, frente a un incremento promedio anual de 2.000 la década anterior. Además, se observó una reducción en las prácticas de aislamiento y sujeción mecánica en estas instituciones. Los cambios afectaron fundamentalmente a personas con esquizofrenia y se dieron de forma abrupta. Esto se atribuía al uso de neurolépticos. 
Esta conclusión fue criticada, porque no se podía establecer relación causal entre estos sucesos. Ello requería al menos un análisis de cohortes comparando resultados entre expuestos y no expuestos a neurolépticos. El estudio de Epstein et al. (18) analizó las tasas de altas en los frenopáticos de California, en función de si los sujetos habían sido tratados o no con ataráxicos y de si el hospital practicaba o no un uso intensivo de estos. Estudiaron a los varones blancos de 24 a 44 años que ingresaron por primera vez en 1956 y 1957. En 1956, se produjeron 673 ingresos, el 36\% recibió ataráxicos durante los seis primeros meses $y$, de estos, el $63 \%$ fue dado de alta a los 6 meses y el $74 \%$ a los 18 meses, frente al $67 \%$ y el $88 \%$ respectivamente del grupo que no los recibió. En 1957 se produjeron 740 ingresos, el 48\% recibieron ataráxicos, de estos, al final del sexto mes, el $64 \%$ había sido dado de alta, frente al $71 \%$ de los que no lo habían recibido. Cuando se compararon los tres hospitales con mayor utilización de fármacos con los tres de menor consumo, no se hallaron diferencias en la tasa de retención hospitalaria total en 1956, y fue algo más alta en 1957 en los primeros. El porcentaje de pacientes tratados con ataráxicos en el conjunto de hospitales de uso elevado fue del $49 \%$ en 1956, y del $63 \%$ en 1957; estas cifras para los hospitales de bajo uso fueron del 26\% en 1956, y del 34\% en 1957.

Los primeros ensayos con clorpromazina, no controlados, produjeron valoraciones muy positivas, pero pronto se reconoció que el entusiasmo debía refrenarse. No había Ilegado la "penicilina psiquiátrica". Con los primeros ensayos controlados con placebo y diseño cruzado quedaron claras las indicaciones estrictas de clorpromazina: estados de aumento de excitación y tensión. Controlaba cualquier estado grave que cursara con hipermotilidad e iniciativa anormal aumentada, pero era incapaz de modificar delirios y alucinaciones. Se llamaba a los neurolépticos "camisas de fuerza" y "lobotomizadores" químicos (14).

En los primeros estudios comparativos, los neurolépticos se mostraron superiores a sus antecesores barbitúricos. Un estudio (19) realizado con 641 pacientes esquizofrénicos varones en su primer ingreso, aleatorizado y a doble ciego, de 12 semanas, comparó cinco ramas de tratamiento con neurolépticos (clorpromazina, triflupromazina, mepazina, proclorperazina y perfenazina) entre sí y contra fenobarbital. Los tratamientos se iniciaron a dosis equivalentes, que se incrementaron protocolizadamente y luego fueron ajustadas con criterio clínico. Emplearon 24 criterios de cambio clínico. La dosis diaria media de clorpromazina fue de $635 \mathrm{mg}$ y, de perfenazina, de 50 mg. Finalizaron el ensayo el $74 \%$ de los sujetos. Solo 21 abandonaron por reacciones adversas. Los cinco neurolépticos se mostraron mejores que fenobarbital y todos fueron igualmente efectivos, excepto mepazina que fue inferior al resto. Los criterios de cambio que más mejoraron fueron: resistencia, beligerancia, alteración del pensamiento, y gravedad de la enfermedad.

\section{¿Sin neuroléptico, no hay tratamiento?}

El estudio de Cole et al. (20) comparó el efecto de tres neurolépticos (clorpromazina, flufenazina y tioridazina) contra placebo en un ensayo clínico con asignación aleatoria y diseño paralelo, de seis semanas, en el que participaron 463 sujetos con esquizofrenia aguda de hospitalización reciente. Las dosis se ajustaron con criterio clínico flexible resultando una dosis diaria media equivalente a 650 mg de clorpromazina. La valoración fue realizada por psiquiatras y enfermeras. Completaron el estudio el $74 \%$ y la mayoría de los abandonos prematuros por falta de eficacia procedían del grupo placebo. Ningún sujeto tratado con neurolépticos empeoró, un $5 \%$ se mantuvo estable, el 20\% mejoró mínimamente, y el $75 \%$ restante mejoró "mucho o muchísimo". Con placebo el $40 \%$ mejoró "mucho o muchísimo". De un conjunto de 21 factores, los 13 que mejoraron significativamente más con neurolépticos que con placebo fueron: participación social, confusión, cuidado de sí mismo, síntomas hebefrénicos, agitación-tensión, conversación lenta, conversación incoherente, irritabilidad, indiferencia al medio ambiente, hostilidad, alucinaciones auditivas, ideas persecutorias, y desorientación. El efecto de los neurolépticos trascendía la mera tranquilización.

En el proyecto anterior había una segunda parte (21), centrada en el ajuste en la comunidad de los individuos que hubieran sido dados de alta. De los 344 sujetos que completaron el ensayo $299(87 \%)$ fueron dados de alta, de estos 176 
(59\%) no precisaron rehospitalización en el año siguiente, y de los 123 que fueron rehospitalizados, 78 volvieron a ser dados de alta; de modo que al año de estudio estaban ubicados en la comunidad 254 sujetos. El $68 \%$ de estos presentaban psicopatología mínima o ausente. Solo el 11\% mostraba un ajuste social equiparable al de la población general, pero la mayoría (68\%) había vuelto a su mejor nivel funcional previo, y al 57\% se los consideraba como personas activas o moderadamente activas. Entre los asalariados reales o potenciales, el $12 \%$ no había Ilegado a estar empleado en el año, el $58 \%$ estaba empleado al final del periodo de seguimiento; de estos, el $68 \%$ presentaba un trabajo conforme a su nivel formativo y el $54 \%$ era económicamente autosuficiente. El 64\% de las amas de casa se estaban desempeñando adecuadamente. Cuando se trató de determinar la influencia de diversos factores personales, premórbidos y del tratamiento sobre el ajuste social se encontró que los sujetos en el grupo placebo tuvieron la menor probabilidad de rehospitalización. En los sujetos que recibieron neurolépticos hubo una relación positiva entre mejoría al alta y ausencia de psicopatología al año del alta. El 80\% de los que nunca recibieron neurolépticos o los utilizaron continuadamente tras el alta asistieron con regularidad al trabajo; pero esto ocurrió solo en el $56 \%$ de los que tomaron fármacos solo en algunos momentos.

En otro estudio (22) 80 sujetos con esquizofrenia, con edades entre 16 y 40 años, la mayoría con una o ninguna hospitalización previa, fueron aleatorizados para recibir a doble ciego, placebo o clorpromazina (hasta 900 mg/día). En el momento del alta los pacientes con clorpromazina se mostraron significativamente mejor que los sujetos con placebo. Tras el alta fueron seguidos durante tres años. El tratamiento ambulatorio no fue controlado por el equipo investigador, así que en el análisis final de resultados se agruparon los sujetos en cuatro categorías, según el resultado de la aleatorización y del uso de neurolépticos durante el seguimiento: 24 sujetos en grupo PL-Off (tomaron placebo en hospital, y no tomaron neurolépticos ambulatoriamente), 17 en el grupo PL-On (tomaron placebo en la hospitalización pero recibieron neurolépticos durante el seguimiento), 22 sujetos en CPZ-On (aleatorizados a clorpromazina y continuaron con neurolépticos durante el seguimiento) y 17 sujetos en CPZ-Off (aleatorizados a clorpromazina, pero sin neurolépticos tras el alta). Al final del seguimiento, 39 sujetos estaban tomando neurolépticos y lo hicieron regularmente al menos 2/3 del tiempo; y 41 no tomaban neurolépticos ni lo hicieron al menos durante $2 / 3$ del tiempo. El grupo PL-Off mostró una mejoría significativamente superior a los otros tres, no hubo diferencias entre PL-On y CPZ-On. Requirieron rehospitalización el $8 \%$ de los sujetos del grupo PL-Off, el $73 \%$ del grupo CPZ-On, el 53\% del grupo PL-On y el $47 \%$ del CPZ-Off.

Posteriormente, un ensayo aleatorizado comparó cinco formas de tratamiento: terapia del medio (milieu therapy) sola o asociada a psicoterapia psicoanalítica, a neurolépticos, a psicoterapia más neurolépticos, o asociada a terapia electroconvulsiva (23). El seguimiento se prolongó hasta cinco años. Los grupos tratados con neurolépticos o terapia electroconvulsiva obtuvieron mejores resultados inicialmente, pero esta superioridad se disipó al cabo de tres años.

Los estudios referidos muestran una superioridad a corto plazo de los neurolépticos sobre placebo en el tratamiento de episodios agudos de esquizofrenia. Esto ha sido confirmado con metaanálisis (24).

La duración de la psicosis sin tratar afecta negativamente la respuesta posterior a los neurolépticos y se propuso que, de rutina, todos los casos se trataran inmediatamente, dado que se consideraba que demorar el inicio del tratamiento para dar tiempo a remisiones expontáneas (trastornos esquizofreniformes) sería lesivo para el paciente y, por tanto, lo ético era iniciar el tratamiento sin demora, con la aparición de los primeros síntomas. Se realizó un metaanálisis (25) que incluyó estudios comparativos con asignación aleatoria o cuasiexperimentales, de sujetos en su primer o segundo episodio esquizofrénico, con al menos un grupo control sin neurolépticos y seguimiento de un año o más. Se excluyeron los estudios en los que se le retiraba a un grupo el tratamiento antipsicótico previo. Se encontraron seis estudios, con un total de 623 sujetos. No se evidenció daño relacionado con las terapias sin neurolépticos, incluso cuando se analizaron solo los estudios aleatorizados.

La esquizofrenia normalmente presenta un periodo prodrómico de uno a tres años, con conductas y síntomas psicológicos inespecíficos, deterioro 
funcional y breves manifestaciones psicóticas atenuadas e intermitentes. Entre el $22 \%$ y el $40 \%$ de las personas con estas características desarrollan un episodio psicótico franco antes de un año y están en ultra-elevado riesgo de transición a psicosis. Prevenirlo o retrasarlo podría ser de utilidad. Una revisión reciente (26) incluyó los estudios aleatorizados, que evaluaron el efecto de cualquier intervención, para prevenir la transición a psicosis en personas con síntomas prodrómicos pero sin episodios previos. De 11 estudios incluidos, en cinco intervenían neurolépticos (risperidona, olanzapina y amisulpride).

Se concluyó que los neurolépticos no han mostrado efecto en la prevención del inicio de psicosis, aunque sí lo lograron algunas terapias psicológicas complejas.

\section{¿Previenen las recaídas?}

Se recomienda tratamiento neuroléptico continuado indefinidamente para evitar recaídas en la esquizofrenia (2). Un metaanálisis reciente (27) que incluyó 65 ensayos clínicos, un total de 6.493 sujetos, avala este uso. Después de un periodo de estabilidad, grupos que continuaban el tratamiento neuroléptico se compararon con otros a los que se les sustituía por placebo. Las recaídas al año fueron del $27 \%$ en los grupos con neuroléptico, frente al $69 \%$ con placebo, las rehospitalizaciones fueron del $10 \%$ y del $26 \%$, respectivamente. Los grupos con fármaco presentaron más efectos adversos que los grupos placebo. La duración media y la moda de los ensayos fueron de 26 semanas, y muy pocos duraron 1 año, solo uno alcanzó los 2 años. Una metarregresión encontró que, a mayor duración del seguimiento, la diferencia en la frecuencia de recaídas entre el grupo placebo y antipsicótico disminuía, hasta anularse tras dos años de seguimiento. En los estudios incluidos, la retirada del neuroléptico de estabilización para sustituirlo por placebo, en general, fue brusca, y aunque en 11 se decía gradual, 28 días de promedio, se considera rápido. En los grupos placebo hubo una mayor incidencia de discinesia, un síntoma por retirada de neurolépticos. En algunos pacientes, luego de entre cuatro y seis semanas de la retirada de neurolépticos utilizados crónicamente, aparece un cuadro de empeoramiento de síntomas positivos, con agitación y acatisia, acompañado de manifestaciones vegetativas $y$, generalmente, precedido por discinesias (28). Por tanto, más que probar la utilidad del mantenimiento en la prevención de recaídas, lo que muestra es un aumento del riesgo de psicosis por la retirada, es decir, no un efecto terapéutico, sino un efecto tóxico.

Un estudio de 2 años (29), con sujetos de 18 a 45 años en primer episodio psicótico, trataba de determinar cómo influía la reducción-retirada temprana del neuroléptico, en comparación con el mantenimiento convencional, sobre el riesgo de recaída y ajuste sociolaboral. Se reclutaron 131 sujetos que, tras seis meses de remisión de síntomas, fueron aleatorizados y seguidos de forma abierta durante 18 meses. Completaron 128 sujetos, recayeron el $43 \%$ en el grupo de reducción-retirada y el $21 \%$ en el de mantenimiento. No hubo diferencias en los resultados funcionales. Todos los que completaron el estudio fueron invitados a continuar el seguimiento hasta siete años (30). Durante este seguimiento prolongado, el tratamiento lo estableció el equipo clínico, no los investigadores. La variable principal de resultado fue la tasa de recuperación, definida con criterios sintomáticos y funcionales, según el grupo asignado en el estudio inicial. A los siete años, se localizaron 103 sujetos, 51 del grupo mantenimiento y 52 del de reducción-retirada. Los sujetos perdidos no mostraron diferencias con los localizados. La tasa de recuperación en ese momento fue del $17,6 \%$ con mantenimiento, y del $40,4 \%$ con reducción-retirada de dosis. La mayor tasa de recuperación en el grupo de reducción-retirada del antipsicótico se debe a un mejor estado funcional, mientras que el estado sintomático fue similar en ambos grupos. Tampoco hubo diferencias en las reagudizaciones. Las curvas de supervivencia de ambos grupos se aproximaban hasta cruzarse al tercer año. En total, 67 sujetos tuvieron como mínimo una recaída en los siete años de seguimiento, 35 estaban con mantenimiento, y 32 con reducción-retirada. Hubo 36 que no sufrieron ninguna recaída, 20 estaban en el grupo de reducción-interrupción y 16 en el grupo con mantenimiento.

Un estudio de seguimiento a 20 años (31) pretendió averiguar con qué frecuencia y gravedad experimentan psicosis las personas con esquizofrenia mantenidas con neurolépticos, y si es menos grave que cuando no se utilizan. Para ello se reclutó una cohorte de 139 sujetos (70 con 
esquizofrenia y 69 para el grupo control con trastorno psicótico del humor) que fueron evaluados prospectivamente durante una hospitalización por agudización al inicio de su enfermedad, y reevaluados a 2; 4,5; 7,5; 10; 15 y 20 años. Procedían de sucesivas admisiones en dos hospitales. Los entrevistadores desconocían los diagnósticos, los resultados de evaluaciones previas y la finalidad del estudio. La fiabilidad interevaluadores fue satisfactoria. En el grupo con esquizofrenia, en los momentos de evaluación, entre el $62 \%$ y el $67 \%$ de los sujetos recibían neurolépticos, entre el $3 \%$ y el $14 \%$ recibían otros fármacos psicoactivos pero no neurolépticos, y entre el 23 y el $31 \%$ no recibieron psicofármacos. La mediana de las dosis antipsicóticas a los 10 y 15 años fue equivalente a 575 mg y 500 mg de clorpromazina, respectivamente. Cuando se hicieron todas las evaluaciones, 25 sujetos estaban recibiendo neurolépticos (grupo 1); 24 en algunas, pero no en todas (grupo 2); y 15 no recibieron neurolépticos en ninguna evaluación (grupo 3). De seis sujetos se dispuso de datos en menos de cuatro evaluaciones. En las evaluaciones, entre el $56 \%$ y el $78 \%$ de los sujetos que estaban tomando neurolépticos presentaron actividad psicótica, frente al 17\% y el $27 \%$ que no los consumían. Además, mientras que en el grupo 1, del $68 \%$ al $86 \%$ presentaban actividad psicótica, en el grupo 3 lo hacían entre el $7 \%$ y el $60 \%$. Los síntomas psicóticos causaron alteraciones de la vida social y la capacidad instrumental, de moderadas a graves, entre el 35\% y el $73 \%$ de los sujetos del grupo 1 , y el $0 \%$ y el $30 \%$ en el grupo 3. Las diferencias entre grupos con y sin neurolépticos alcanzaron significación estadística a partir de la evaluación a los 4,5 años. Presentaron síntomas psicóticos en al menos cuatro evaluaciones el $72 \%$, el $46 \%$ y el $7 \%$ de los sujetos de los grupos 1,2 , y 3 , respectivamente. Solo 12 sujetos con esquizofrenia estuvieron libres de psicosis en todas las evaluaciones de seguimiento; siete de ellos pertenecían al grupo 3; los otros cinco al grupo 2. Se definió recuperación completa como la ausencia de síntomas positivos y negativos y de rehospitalización, la existencia de algunos contactos sociales, y trabajo durante al menos la mitad del tiempo, en el año previo a la evaluación. Dos sujetos del grupo 3 estuvieron en recuperación completa en todas las evaluaciones. La mitad de los sujetos del grupo 1 estuvo libre de síntomas psicóticos en al menos una de las evaluaciones de seguimiento, indicando potencial para recuperación.

Entre los controles con psicosis afectiva, se observaron resultados similares. En la última evaluación, el 28\% recibía neurolépticos y el 37\% recibía otros medicamentos pero no neurolépticos. Solo el $12 \%$ presentó actividad psicótica en más de tres evaluaciones y la proporción de sujetos con actividad psicótica a los 7,5 y 10 años fue mayor en los usuarios de neurolépticos.

Al menos un grupo de personas con esquizofrenia puede prescindir a largo plazo del uso continuado de neurolépticos. Puede que el estado de desinterés e indiferencia emocional, beneficioso en el episodio agudo, sea nocivo a largo plazo para la recuperación personal, social e instrumental del sujeto.

\section{¿Son medicamentos tolerados y seguros?}

Es frecuente que los ensayos clínicos con neurolépticos informen solo de efectos adversos que aparecen con frecuencia $\geq 5 \%$, e incluso $\geq 10 \%$. Efectos no muy frecuentes pero graves pueden pasar desapercibidos. Hay dudas de que se informe siempre, como es preceptivo, sobre la frecuencia del resultado "muerte". El resultado "pérdidas debidas a acontecimientos adversos" suele mezclar efectos adversos y falta de respuesta como exacerbación de la psicosis, con lo que no es un buen indicador de tolerabilidad-seguridad, sino mixto de tolerabilidad-eficacia. Una proporción importante de personas tratadas con neurolépticos padecen efectos adversos $(24,27)$, que afectan su calidad de vida.

En un año desarrollan pseudoparkinsonismo del $37 \%$ al $44 \%$, acatisia del $26 \%$ al $35 \%$; discinesia tardía del 1,1 al 4,5\% (32), la prevalencia de discinesia aumenta con el uso continuo. Los efectos adversos anticolinérgicos también son muy frecuentes: en ensayos clínicos padecieron visión borrosa del $10 \%$ al $20 \%$, otro tanto estreñimiento, sequedad de boca del $5 \%$ al $33 \%$, dificultades urinarias el 10\%, hipersalivación y babeo del $4 \%$ al $42 \%$; centralmente interfieren con las capacidades cognitivas (33). Eran obesos el 33\% de los pacientes hospitalizados que tomaban neurolépticos, el $68 \%$ presentó dislipemia, el $64 \%$ 
hipercolesterolemia y el $30 \%$ hipertrigliceridemia (34). Se detectó hiperprolactinemia hasta en el $42 \%$ de varones y el $75 \%$ de mujeres con neurolépticos, lo que conduce a ginecomastia, galactorrea, irregularidades menstruales, infertilidad, disfunción sexual, atrofia gonadal, acné e hirsutismo femenino, a largo plazo posiblemente osteoporosis, fracturas óseas y cáncer de mama (35). Se produce enuresis entre el $6 \%$ y el $20 \%$ de usuarios (36). Convulsionan el $2,7 \%$ de sujetos con clozapina y en un $0,8 \%$ aparece agranulocitosis. Tienen somnolencia el 39\% de los tratados con olanzapina, y más frecuentemente con otros neurolépticos (37). Se detecta prolongación anómala del intervalo QTc del electrocardiograma (marcador de riesgo de muerte súbita) en el $8 \%$ de sujetos (38). El tromboembolismo pulmonar también se ha asociado con el uso de neurolépticos (39).

La mayoría de los efectos adversos son dosis dependiente y se potencian con politerapia. La polifarmacia y megadosis neuroléptica son frecuentes, por el uso de otros psicofármacos para la comorbilidad psiquiátrica, y de otros medicamentos prescriptos para problemas de salud física o para corregir efectos adversos. La polimedicación conlleva más riesgo de interacciones y adversidades. Los médicos tienden a subestimar, en comparación con la percepción de los usuarios, la frecuencia y gravedad de los efectos adversos por neurolépticos, prácticamente en todas las áreas: psíquica, neurológica, autonómica y otras (40).

Las anomalías en la anatomía cerebral identificadas en la esquizofrenia y su progresión en el tiempo pueden ser causa (hacen al paciente susceptible) o consecuencia del trastorno (se agravan con la presión psicológica y el aislamiento), o también efecto del tratamiento (efecto tóxico del neuroléptico). Se siguieron prospectivamente 211 sujetos desde su primer episodio psicótico hasta 14 años (media 7,2 años), y se realizaron entre dos y cinco exploraciones de imagen cerebral mediante resonancia magnética. Se trató de determinar la contribución de cuatro potenciales predictores de pérdida de volumen cerebral: duración y gravedad de la enfermedad, tratamiento neuroléptico y abuso de sustancias. La reducción generalizada y específica de tejido cerebral se asoció a la intensidad del tratamiento neuroléptico después de controlar el efecto de los otros tres predictores. A más tratamiento neuroléptico mayor pérdida de materia gris. La gravedad de la enfermedad tuvo un efecto modesto y el abuso de sustancias no presentó una asociación significativa, después de controlar los otros predictores (41). Posteriormente se ratificó la importancia de la intensidad del tratamiento antipsicótico y se añadió la duración de las recaídas como otra variable que significativamente predice la pérdida de tejido cerebral (42), aunque "recaída" se definió después de la recogida de datos. Un metaanálisis asoció la merma longitudinal de materia gris en personas con esquizofrenia con la exposición acumulada a neurolépticos a lo largo del tiempo, pero no con cambios en la sintomatología, ni con la duración de la enfermedad (43).

Las personas con esquizofrenia sufren un exceso de mortalidad (44). En el periodo 1999-2008 la mortalidad para los diagnosticados de esquizofrenia fue del $20 \%$ y del $9,37 \%$ para los no diagnosticados. Este exceso afectaba a los más jóvenes, atenuándose con la edad, hasta anularse para los nonagenarios. La diferencia se mantuvo para todas las causa de muerte, excepto cáncer en conjunto. Aunque la mortalidad por cáncer de pulmón fue mayor entre esquizofrénicos. El exceso de mortalidad se mantuvo después de realizar ajustes. Esto se ha atribuido al trastorno mental, las dificultades de autocuidado, sedentarismo, tabaquismo, abuso de sustancias, y los efectos adversos de los neurolépticos.

Algunos (45) han encontrado que el riesgo de muerte en esquizofrénicos fue menor para los que utilizaban neurolépticos hasta su deceso, respecto de los que no los recibían en ese momento. También encontró que el riesgo de muerte fue significativamente menor en los sujetos con uso continuado de neurolépticos que en aquellos que nunca los tomaron extrahospitalariamente. Hubo una relación inversa entre mortalidad y duración acumulada de uso de neurolépticos. No obstante, la tasa de mortalidad fue especialmente baja para aquellos cuya duración acumulada de uso de neurolépticos fue menor a 6 meses, no se contabilizaron las muertes intrahospitalarias, a los pacientes con salud muy debilitada se les suelen retirar los neurolépticos, y los incumplidores pueden presentar peor salud por otras razones. 


\section{DESFASE ENTRE EVIDENCIA Y PRÁCTICA}

Se han desarrollado recomendaciones y guías para ayudar a la toma de decisiones terapéuticas en la esquizofrenia (2) basadas en la mejor evidencia disponible. El uso de neurolépticos apegado a las guías se ha asociado a una mayor reducción de la psicopatología y a menores efectos adversos (46). Sin embargo, su seguimiento parece la excepción más que la regla (47). Puede que los ensayos clínicos tengan un diseño defectuoso, o exista sesgo de publicación o de selección de los pacientes.

El 64\% de 2.000 ensayos clínicos para esquizofrenia publicados entre 1948 y 1997 eran de baja calidad y solo el $1 \%$ logró la puntuación máxima. Solo el 4\% describió adecuadamente el método de asignación a los grupos control y experimental. Solo el 22\% describió el método de cegado y solo el $42 \%$ lo ocurrido con las pérdidas. Solo uno discutió adecuadamente el problema de la potencia estadística, y solo 3 tuvieron suficiente tamaño de muestra para detectar una diferencia del $20 \%$, con una significación del $5 \%$ y una potencia del $80 \%$. El 50\% tuvo menos de 50 participantes. El 50\% duró $\leq 6$ semanas, solo el $19 \%$ duró > 6 meses. El 86\% evaluó fármacos; el $63 \%$ midió, como resultado principal, cambios sintomáticos, empleándose 640 escalas diferentes; el $20 \%$ valoró la sintomatología global. Variables con significado clínico directo como "actividades de la vida diaria" o "funcionamiento global" se evaluaron en el $4 \%$ y el $6 \%$, respectivamente. Solo el $22 \%$ evaluó efectos adversos. El $91 \%$ de los ensayos se realizaron en EE.UU. o Europa (48).

Una década después había 10.000 ensayos clínicos sobre esquizofrenia (49), pero su accesibilidad era reducida, solo el $28 \%$ estaba disponible en PubMed. Aumentó a 60 la mediana de participantes por ensayo, pero también la publicación duplicada de estudios. Actualmente el $25 \%$ de los ensayos proceden de China. No se han producido otros cambios.

En la información para solicitud de comercialización de ocho neurolépticos se localizaron 24 ensayos clínicos precomercialización, de los cuales 20 estaban publicados, el tamaño del efecto publicado fue, en promedio, un $8 \%$ superior al indicado en los documentos de aprobación. De los cuatro restantes, tres no mostraron superioridad frente al placebo y otro no mostró superioridad frente al comparador activo (50).

En los ensayos clínicos de neurolépticos para la esquizofrenia, suele excluirse a los sujetos con tendencias suicidas o abuso de sustancias, lo que podría restar validez externa a sus resultados, al seleccionar un tipo de sujeto distintos de los vistos en la asistencia rutinaria. Lo mismo puede decirse respecto de la disposición a participar. Pero cuando en un estudio que no contempló estos criterios de exclusión, compararon los resultados a los 12 meses en función de si los sujetos presentan o no estas comorbilidades, no hallaron diferencias en cuanto al tiempo hasta la interrupción del tratamiento, pérdidas, psicopatología o funcionamiento social, aunque los primeros sí mostraron más tendencias depresivas y tardaron menos en ser rehospitalizados, diferencias que se asociaron al abuso de sustancias y no a las tendencias suicidas (51). Cuando se compararon las características de los sujetos que participaron en un ensayo con neurolépticos, con las de no participantes que cumplían los criterios de inclusión/no-exclusión, tampoco se detectaron diferencias, salvo que estos últimos tenían más probabilidad de haber estado hospitalizados por motivos físicos en el año previo (52). Estas diferencias implican un uso de neurolépticos más cuidadoso.

Con el tiempo, se ha producido una progresiva disminución de la diferencia de eficacia entre placebo y neurolépticos en los ensayos clínicos realizados entre 1991 a 2006, debido al aumento del efecto del placebo y la disminución del efecto del neuroléptico, incluso como controles activos. Esto ha ocurrido a pesar del aumento del número de sujetos, la disminución de pérdidas, el mantenimiento de la gravedad basal del trastorno, y la mejora de la calidad de los estudios (53). En este contexto, la realización de ensayos controlados con placebo se impone éticamente, contra la propuesta de emplear solo control activo en los ensayos de neurolépticos para la esquizofrenia.

\section{¿Qué sienten las personas que toman neurolépticos?}

La mitad de usuarios de neurolépticos han experimentado síntomas de retirada (54), que aparecen al dejar el tratamiento, algunos son similares 
a los que motivaron la prescripción, y otros nuevos, de intensidad y momento de aparición variable según ciertas características farmacológicas, la forma de utilización y la persona que lo ha recibido. Frecuentemente, son interpretados como prueba de la persistencia del trastorno inicial y necesidad de continuar el tratamiento. Después, pacientes y médicos suelen ser reticentes a nuevas retiradas, temen la "recaída". Otros pacientes persisten en abandonar el neuroléptico, en general, por percibir que los beneficios no compensan los efectos adversos (55).

En los ensayos clínicos con neurolépticos, las tasas de abandono globales son del $33 \%$ al $42 \%$ $(24,27)$. En condiciones clínicas rutinarias aproximadamente el $40 \%$ de los pacientes incumplen el tratamiento neuroléptico antes de un año, y el 75\% antes de dos. Se considera que hay falta de adherencia terapéutica cuando el sujeto toma menos del $80 \%$ de la dosis prescrita, pero solo el $17 \%$ de los sujetos toma 1/5 o menos de las dosis neurolépticas prescritas (56). La prescripción de neurolépticos inyectables de larga duración es la estrategia más utilizada para mejorar el cumplimiento. Su empleo se basa en resultados de estudios en "espejo" que comparan las recaídas antes y después de su aplicación, siendo cada paciente su propio control (57), diseño con elevado riesgo de sesgos. En ensayos clínicos aleatorizados con control paralelo, no han mostrado ser más efectivos ni seguros que los neurolépticos orales (58).

El tratamiento ambulatorio obligatorio, administrado por orden judicial contra la voluntad del sujeto, tampoco parece resolver el problema, también se apoya en estudios "en espejo", mientras que estudios aleatorizados con control paralelo no muestran beneficios adicionales (59).

Como consecuencia de las respuestas coercitivas a las quejas sobre el tratamiento y los intentos de abandono del neuroléptico, el sujeto realiza cambios en el tratamiento que le permiten ejercer su autonomía pero que oculta, por lo que el terapeuta maneja datos falsos. Cuando sucede, se considera que falta conciencia de enfermedad. Si aparecen síntomas, estos se consideran "refractarios" y se recurre a "megadosis", politerapia y coacción, que forman una espiral ascendente de efectos desagradables y desestabilización del sujeto. Finalmente, una ruptura de la relación terapéutica, o un desenlace más trágico, "legitima" al entorno para forzar tratamientos hasta límites irracionales e inmorales. Por el contrario, la implicación del sujeto en su tratamiento, con escucha atenta a las dificultades que experimente, buscando concordancia de objetivos con el paciente, sería una respuesta más adecuada. Incluso ante el deseo de un sujeto de abandonar completamente la medicación neuroléptica, la aplicación de una política de reducción de daños evitaría el abandono abrupto del tratamiento con pérdida de seguimiento del paciente, además permitiría el ofrecimiento de alternativas (60).

Los neurolépticos son, sobre todo, fármacos psicoactivos y producen un estado físico y mental alterado e impredecible, que interacciona con la experiencia de disgusto y discapacidad que había inducido al sujeto a pedir ayuda. Puede ser útil si conlleva la supresión de alguna de sus quejas. Este efecto inespecífico puede reflejarse en las escalas de valoración de síntomas. Los ensayos clínicos están focalizados en un estrecho conjunto de quejas y resultados y relegan a la categoría de efectos colaterales al resto, lo que dificulta la identificación del rango completo de efectos psicoactivos y físicos de los neurolépticos. También hay lagunas sobre sus efectos a largo plazo, incluyendo los de retirada.

Algunos estudiosos describen el efecto de los neurolépticos como "una petrificación en las emociones, bloqueando la iniciativa de la persona", "su curiosidad e iniciativa intelectual se transforman en actitudes flemáticas y robotizadas", "neutralidad emocional sin trastornos de la conciencia", "camisas de fuerza psíquicas" (61). Al preguntar a jóvenes a los que se les había prescrito neurolépticos, pero que aún no los habían experimentado, sobre sus expectativas, las respuestas fueron: "mucha ayuda para eliminar mis pensamientos y hacer que los síntomas no me molesten"; pero cuando se les volvió a preguntar tras seis semanas de su uso continuado, dijeron que el neuroléptico les había producido un estado de "indiferencia y desapego sobre los síntomas" (62). En una base de datos de acceso libre en Internet, en la que los usuarios de neurolépticos relataban sus experiencias, los efectos subjetivos predominantemente descritos fueron sedación, discapacidad cognitiva, y aplanamiento o indiferencia afectiva (63). Esta información es coherente con el papel de la dopamina en los procesos asociados con la capacidad de experimentar placer y motivación. 


\section{¿Cómo se toman las decisiones terapéuticas?}

A los asistentes a un congreso de psiquiatría se les presentó un caso de esquizofrenia en su segunda recaída y se les preguntó si prescribirían un neuroléptico depot o continuarían con el tratamiento oral. Mayoritariamente recomendaron la primera opción. Luego, con el mismo caso y opciones, el paciente les peguntaba ¿qué haría usted en mi lugar?, la respuesta fue la misma. Finalmente, se les dijo que imaginaran que eran ellos mismos los protagonistas del caso, entonces la respuesta cambió, la mayoría eligió continuar con el oral (64).

En otra ocasión se presentaron dos casos. En uno, un paciente debe ser tratado con uno de dos nuevos neurolépticos sobre los que se proporciona información en una lista de 12 ítems (seis beneficios y seis riesgos) y se los invitó a solicitar información adicional. En el otro, un paciente esquizofrénico va a iniciar tratamiento con un antipsicótico depot, del que se ofrece una lista de 10 propiedades (cinco beneficios y cinco riesgos) y se le pregunta al psiquiatra ¿cuál de estos ítem pasaría al paciente? En el primer caso los psiquiatras solicitaron significativamente más información sobre riesgos para informarse a sí mismos; pero para informar a los pacientes, la situación se invierte y enfatizaron los beneficios (65).

\section{CONCLUSIONES}

De lo expuesto en este trabajo podríamos concluir que:

1) Los datos en los que se apoya la recomendación del uso continuo de fármacos neurolépticos en el tratamiento de la esquizofrenia distan de ser adecuados para sostenerla. Debe mejorarse la calidad de la investigación sobre este punto, en transparencia, tamaño de muestra y duración del seguimiento.

2) Durante la asistencia, la mejora del cumplimiento terapéutico debiera ir de la mano de la capacitación y participación activa del sujeto en su tratamiento. Los métodos coercitivos al uso no son aceptables, independientemente de otras consideraciones éticas, por su falta de eficacia.

\section{REFERENCIAS BIBLIOGRÁFICAS}

1. van Os J, Kapur S. Schizophrenia. Lancet. 2009;374(9690):635-645.

2. Barnes TR, Schizophrenia Consensus Group of British Association for Psychopharmacology. Evidence-based guidelines for the pharmacological treatment of schizophrenia: recommendations from the British Association for Psychopharmacology. Journal of Psychopharmacology. 2011;25(5):567-620.

3. Howes OD, Kapur S. The dopamine hypothesis of schizophrenia: version III - the final common pathway. Schizophrenia Bulletin. 2009;35(3):549-562.

4. Andreasen NC, Carson R, Diksic M, Evans A, Farde L, Gjedde A, Hakim A, Lal S, Nair N, Sedvall G, Tune L, Wong D. Workshop on schizophrenia, PET, and dopamine D2 receptors in the human nestriatum. Schizophrenia Bulletin. $1988 ; 14: 471-484$.
5. Seeman P. All roads to schizophrenia lead to dopamine supersensitivity and elevated dopamine D2(high) receptors. CNS Neuroscience \& Therapeutics. $2011 ; 17(2): 118-132$.

6. Krabbendam L, Hooker CL, Aleman A. Neural effects of the social environment. Schizophrenia Bulletin. 2014;40(2):248-251.

7. Cosgrove L, Krimsky S. A comparison of DSM-IV and DSM-5 panel members' financial associations with industry: a pernicious problem persists. Plos Medicine. 2012;9(3):e1001190.

8. Álvarez JM, Colina F. Origen histórico de la esquizofrenia e historia de la subjetividad. Frenia, Revista de Historia de la Psiquiatría. 2011;11(1):7-26.

9. Hegarty JD, Baldessarini RJ, Tohen M, Waternaux C, Oepen G. One hundred years of schizophrenia: a meta-analysis of the outcome literature. American Journal of Psychiatry. 1994;151(10):1409-1416. 
10. Simó Miñana J. Utilización de medicamentos en España y en Europa. Atención Primaria. 2012;44(6):335-347.

11. Stephenson CP, Karanges E, McGregor IS. Trends in the utilisation of psychotropic medications in Australia from 2000 to 2011. The Australian and New Zealand Journal of Psychiatry. 2013;47(1):74-87.

12. Lindsley $\mathrm{CV}$. The top prescription drugs of 2011 in the United States: antipsychotics and antidepressants once again lead CNS therapeutics. ACS Chemical Neuroscience. 2012;3(8):630-631.

13. Alessi-Saverini S, Biscontri RD, Collins DM, Kazyrskyi A, Sareen J, Enns MW. Utilization and costs of antipsychotic agents: a Canadian population-based study, 1996-2006. Psychiatric Services. 2008;59(5):547-553.

14. Lehmann HE, Ban TA. The history of the psychopharmacology of schizophrenia. Canadian Journal of Psychiatry. 1997;42(2):152-162.

15. Diéguez A, Campos R, Huertas R. Breve historia de la psiquiatría. En: López-Muñoz F, Álamo González C. Historia de la psicofarmacología. Buenos Aires: Panamericana; 2005.

16. Committee on Public Health of the New York Academy of Medicine. Report on tranquilizing drugs. Bulletin of the New York Academy of Medicine. 1957;33(4):282-289.

17. Brill H, Patton RE. Analysis of 1955-1956 population fall in New York State mental hospitals in first year of large-scale use of tranquilizing drugs. American Journal of Psychiatry. 1957;114(6):509-517.

18. Epstein LJ, Morgan RD, Reynolds L. An approach to the effect of ataraxic drugs on hospital release rates. American Journal of Psychiatry. 1962;119:36-47.

19. Casey JF, Lasky JJ, Klett J, Hollister LE. Treatment of schizophrenic reactions with phenothiazine derivatives: a comparative study of chlorpromazine, triflupromazine, mepazine, prochlorperazine, perphenazine, and phenobarbital. American Journal of Psychiatry. 1960;117:97-105.

20. The National Institute of Mental Health Psychopharmacology Service Center Collaborative Study Group. Phenothiazine treatment in acute schizophrenia: Effectiveness. Archive of General Psychiatry. 1964;10(3):246-261.

21. Schooler NR, Goldberg SC, Boothe H, Cole JO. One year after discharge: community adjustment of schizophrenic patients. American Journal of Psychiatry. 1967;123(8):986-995.

22. Rappaport M, Hopkins H, Hall K, Belleza T, Silverman J. Are there schizophrenics for whom drugs may be unnecessary or contraindicated? International Pharmacopsychiatry. 1978;13(2):100-111.

23. May PR, Tuma AH, Dixon WJ, Yale C, Thiele DA, Kraude WH. Schizophrenia. A follow-up study of results of five forms of treatment. Archives of General Psychiatry. 1981;38(7):776-784.

24. Leucht S, Cipriani A, Spineli L, Mavridis D, Orey D, Richter F, Samara M, Barbui C, Engel RR, Geddes JR, Kissling W, Stapf MP, Lässig B, Salanti G, Davis JM. Comparative efficacy and tolerability of 15 antipsychotic drugs in schizophrenia: a multiple-treatments meta-analysis. Lancet. 2013;382(9896):951-962.

25. Bola JR. Medication-free research in early episodeschizophrenia: evidenceoflong-term harm? Schizophrenia Bulletin. 2006;32(2):288-296.

26. Stafford MR, Jackson H, Mayo-Wilson E, Morrison AP, Kendall T. Early interventions to prevent psychosis: systematic review and meta-analysis. BMJ. 2013;346:f185.

27. Leucht S, Tardy M, Kamossa K, Heres S, Kissling W, Salanti G, Davis JM. Antipsychotic drugs versus placebo for relapse prevention in schizophrenia: a systematic review and metaanalysis. Lancet. 2012;379(9831):2063-2071.

28. Gardos G, Cole JO. Maintenance antipsychotic therapy: is the cure worse than the disease? American Journal of Psychiatry. 1976;133(1):32-36.

29. Wunderink L, Nienhuis FJ, Sytema S, Slooff CJ, Knegtering R, Wiersma D. Guided discontinuation versus maintenance treatment in remitted first-episode psychosis: relapse rates and functional outcome. Journal of Clinical Psychiatry. 2007;68(5):654-661.

30. Wunderink L, Nieboer RM, Wiersma D, Sytema S, Nienhuis FJ. Recovery in remitted firstepisode psychosis at 7 years of follow-up of an early dose reduction/discontinuation or maintenance treatment strategy: Long-term follow up of a 2-year randomized clinical trial. JAMA Psychiatry. 2013;70(9):913-920.

31. Harrow M, Jobe TH, Faul RN. Does treatment of schizophrenia with antipsychotic medications eliminate or reduce psychosis?: A 20-year multi-follow-up study. Psychological Medicine. 2014;44(14):3007-3016. 
32. Miller DD, CaroffSN, Davis SM, Rosenheck RA, McEvoy JP, Saltz BL, Riggio S, Chakos MH, Swartz MS, Keefe RSE, Stroup TS, Lieberman JA, Clinical Antipsychotic Trials of Intervention Effectiveness (CATIE) Investigators. Extrapiramidal side-effects of antipsychotics in a randomised trial. British Journal of Psychiatry. 2008;193(4):279-288.

33. Ozbilem M, Adams CE. Systematic overview of Cochrane reviews for anticholinergic effects of antipsychotic drugs. Journal of Clinical Psychopharmacology. 2009;29(2):141-146.

34. Newcomer JW, Haupt DW. The metabolic effects of antipsychotic medications. Canadian Journal of Psychiatry. 2006;51(8):480-491.

35. Carvalho MM, Góis C. Hiperprolactinémia em doentes psiquiátricos. Acta Médica Portuguesa. 2011;24:1005-1012.

36. Harrison-Worlrych M, Skegg K, Ashton J, Herbison $\mathrm{P}$, Skegg DCG. Nocturnal enuresis in patients taking clozapine, risperidone, olanzapine and quetiapine: comparative cohort study. British Journal of Psychiatry. 2011;199(2):140-144.

37. Collaborative Working Group on Clinical Trial Evaluations. Adverse effects of the atypical antipsychotics. Journal Clinical Psychiatry. 1998;59(Suppl 12):S17-S22.

38. Reilly JG, Ayis SA, Ferrier IN, Jones SJ, Thomas SHL. QTC-interval abnormalities and psychotropic drug therapy in psychiatric patients. Lancet. 2000;355(9209):1048-1052.

39. Kamijo Y, Soma K, Nagai T, Kurihara K, Ohwada T. Acute massive pulmonary thromboembolism associated with risperidone and conventional phenothiazines. Circulation Journal. 2003;67(1):46-48.

40. Lindström E, Lewander T, Malm U, Malt UF, Lublin $\mathrm{H}$, Ahlfors UG. Patient-rated versus clinicianrated side effects of drug treatment in schizophrenia. Clinical validation of a self-rating version of the UKU side effect rating scale (UKU-SERS-pat). Nordic Journal of Psychiatry. 2001;55(Suppl 44):5-69.

41. Ho BC, Andreasen NC, Ziebell S, Pierson R, Magnotta $\mathrm{V}$. Long-term antipsychotic treatment and brain volumes: a longitudinal study of firstepisode schizophrenia. Archive of General Psychiatry. 2011;68(2):128-137.

42. Andreasen NC, Liu D, Ziebell BA, Vora A, Ho BC. Relapse duration, treatment intensity, and brain tissue loss in schizophrenia: a prospective longitudinal MRI study. American Journal of Psychiatry. 2013;170(6):609-615.
43. Fusar-Poli P, Smieskova R, Kempton MJ, Ho BC, Andreasen NC, Borgwardt S. Progressive brain changes in schizophrenia related to antipsychotic treatment?: A meta-analysis of longitudinal MRI studies. Neuroscience and Biobehavioral Reviews. 2013;37(8):1680-1691.

44. Kredentser MS, Martens PJ, Chochinov HM, Prior HJ. Cause and rate of death in people with schizophrenia across the lifespan: a populationbased study in Manitoba, Canada. Journal of Clinical Psychiatry. 2014;75(2):154-161.

45. Tiihonen J, Lönnqvist J, Wahlbeck K, Klaukka T, Niskanen L, Tanskanen A, Haukka J. 11-year follow-up of mortality in patients with schizophrenia: a population-based cohort study (FIN11 study). Lancet. 2009;374(9690):620-627.

46. Weinmann S, Hoerger S, Erath $M$, Kilian R, Gaebel W, Becket T. Implementation of a schizophrenia practice guideline: clinical results. Journal of Clinical Psychiatry. 2008; 69(8):1299-1306.

47. Busch AB, Leheman AF, Goldman H, Frank RG. Changes over time and disparities in schizophrenia treatment quality. Medical Care. 2009;47(2):194-207.

48. Thorley B, Adams C. Content and quality of 2000 controlled trials in schizophrenia over 50 years. BMJ. 1998;317:1181-1184.

49. MiyarJ, Adams CE. Content and quality of 10000 controlled trials in schizophrenia over 60 years. Schizophrenia Bulletin. 2013;39(1):226-229.

50. Turner EH, Knoepflmacher D, Shapley L. Publication bias in antipsychotic trials: an analysis of efficacy comparing the published literature to the US Food and Drug Administration Database. Plos Medicine. 2012;9(3):e1001189.

51. Boter H, Derks EM, Fleischhacker WW, Davidson M, Kahn RS, EUFEST Study Group. Generalizability of results of efficacy trials in first-episode schizophrenia: comparisons between subgroups of participants of the European First Episode Schizophrenia Trial (EUFEST). Journal of Clinical Psychiatry. 2010;71(1):58-65.

52. Barnett PG, Scott JY, Rosenheck RA. How do clinical trial participants compare to other patients with schizophrenia? Schizophrenia Research. 2011;130(1-3):34-39

53. Agid O, Siu CO, Potkin SG, Kapur S, Watsky E, Vanderburg D, Zipursky RB, Remington G. Metaregression analysis of placebo response in antipsychotic trials, 1970-2010. American Journal of Psychiatry. 2013;170(11):1335-1344. 
54. Salomon C, Hamilton B. "All roads lead to medication?": Qualitative responses from an Australian first-person survey of antipsychotic discontinuation. Psychiatric Rehabilitation Journal. 2013;36(3):160-165.

55. Dibonaventura M, Gabriel S, Dupclay L, Gupta S, Kim E. A patient perspective of the impact of medication side effects on adherence: results of a cross-sectional nationwide survey of patients with schizophrenia. BMC Psychiatry. 2012;12:20.

56. Remington G, Teo C, Mann M, Hahn M, Fonssias G, Aqid Q. Examining levels of antipsychotic adherence to better understand non-adherence. Journal of Clinical Psychopharmacology. 2013;33(2):261-263.

57. Kishimoto T, Nitta M, Borenstein M, Kane JM, Correll CU. Long-acting injectable versus oral antipsychotics in schizophrenia: a systematic review and meta-analysis of mirror-image studies. Journal of Clinical Psychiatry. 2013;74(10):957-965.

58. Kishimoto T, Robenzadeh A, Leucht C, Leucht S, Wanatabe K, Mimura M, Borenstein M, Kane JM, Correll CU. Long-acting injectable vs oral antipsychotics for relapse prevention in schizophrenia: a meta-analysis of randomized trials. Schizophrenia Bulletin. 2014;40(1):192-213.

59. Kisely SR, Campbell LA, Preston NJ. Compulsory community and involuntary outpatient treatment for people with severe mental disorders review. The Cochrane Database of Systematic Reviews. 2011;(2):CD004408.
60. Aldridge MA. Addressing non-adherence to antipsychotic medication: a harm-reduction approach. Journal of Psychiatric and Mental Health Nursing. 2012;19(1):85-96.

61. Rementerí I. La función de utilidad del uso de las drogas y las culturas de su consumo. Cuadernos Médico Sociales (Chile). 2014;54(1):35-41.

62. Mizrahi R, Bagby RM, Zipursky RB, Kapur S. How antipsychotics work: the patients' perspective. Progress in Neuro-Psychopharmacology \& Biological Psychiatry. 2005;29(5):859-864.

63. Moncrieff J, Cohen D, Mason JP. The subjective experience of taking antipsychotic medication: a content analysis of Internet data. Acta Psychiatrica Scandinavica. 2009;120(2):102-111.

64. Mendel R, Hamann J, Traut-Mattausch E, Bühner M, Kissling W, Frey D. 'What would you do if you were me, doctor?': Randomised trial of psychiatrists personal versus professional perspectives on treatment recommendations. British Journal of Psychiatry. 2010;197(6):441-447.

65. Mendel R, Hamann J, Traut-Mattausch E, Jonas E, Heres S, Frey D, Kissling W. How psychiatrists inform themselves and their patients about risks and benefits of antipsychotic treatment. Acta Psychiatrica Scandinavica. 2009;120(2):112-119.

\section{FORMA DE CITAR}

Pol Yanguas E. Antipsicóticos para la esquizofrenia: paradigma de los medicamentos psiquiátricos. Salud Colectiva. 2015;11(1):115-128.

Recibido: 17 de agosto de 2014

Versión final: 21 de septiembre de 2014

Aprobado: 18 de diciembre de 2014

Este obra está bajo una licencia de Creative Commons Reconocimiento-NoComercial 4.0 Internacional. Reconocimiento - Permite copiar, distribuir y comunicar públicamente la obra. A cambio, se debe reconocer y citar al autor original. No Comercial - Esta obra no puede ser utilizada con finalidades comerciales, a menos que se obtenga el permiso. 